\title{
Consensus of Fractional-Order Double-Integral Multi-Agent System in a Bounded Fluctuating Potential
}

\author{
Xi Chen, Maokang Luo and Lu Zhang *(D) \\ College of Mathematics, Sichuan University, Chengdu 610065, China; chenxi221b@stu.scu.edu.cn (X.C.); \\ makaluo@scu.edu.cn (M.L.) \\ * Correspondence: zhanglucd@scu.edu.cn
}

check for updates

Citation: Chen, X.; Luo, M.; Zhang, L. Consensus of Fractional-Order Double-Integral Multi-Agent System in a Bounded Fluctuating Potential. Fractal Fract. 2022, 6, 147. https:// doi.org/10.3390/fractalfract6030147

Academic Editors: Giacomo Ascione, Alessandra Meoli and Enrica Pirozzi

Received: 13 February 2022

Accepted: 4 March 2022

Published: 7 March 2022

Publisher's Note: MDPI stays neutral with regard to jurisdictional claims in published maps and institutional affiliations.

Copyright: (C) 2022 by the authors. Licensee MDPI, Basel, Switzerland. This article is an open access article distributed under the terms and conditions of the Creative Commons Attribution (CC BY) license (https:// creativecommons.org/licenses/by/ $4.0 /)$.

\begin{abstract}
At present, the consensus problem of fractional complex systems has received more attention. However, there is little literature on the consensus problem of fractional-order complex systems under noise disturbance. In this paper, we present a fractional-order double-integral multiagent system affected by a common bounded fluctuating potential, where the protocol term consists of both the relative position and velocity information of neighboring agents. The consensus conditions of the presented system in the absence of noise are analytically given and verified by a numerical simulation algorithm. Then, the influences of the system order and other system parameters on the consensus of the presented system in the presence of bounded noise are also analyzed. It is found that when compared with the classical integer-order system, the presented fractional-order system has a larger range of consensus parameters and has more rich dynamic characteristics under the action of random noise. Especially, the bounded noise has a promoting effect on the consensus of the presented fractional-order system, while there is no similar phenomenon in the corresponding integer-order system.
\end{abstract}

Keywords: fractional-order system; multi-agent system; consensus; fluctuating potential; bounded noise

\section{Introduction}

Complexity science [1-3] studies complex systems and complexity and reveals and explains the operating laws of complex systems, involving nature, engineering, biology, economics, management, politics, and society. It is known as 'science in the 21st century'. As an important piece of research content of complexity science, due to its important theoretical and practical significance, multi-agent system (MAS) [4,5] has become a new research topic in many fields such as system, control, and artificial intelligence, and has been widely concerned by scholars in many fields.

In the past decades, scholars have carried out in-depth research on the consensus and multi-consensus [6,7] of the first-order and second-order (linear and nonlinear, homogeneous and heterogeneous $[8,9])$ MASs under different topologies, time delays, sampleddata, asynchronous and aperiodic communications, time-driven jumps, and have achieved fruitful results [10-15]. Subsequently, some scholars extended the classical first-order and second-order MASs to high-order MASs [16-19]. Since the beginning of the 21st century, the study of fractional calculus has made notable progress. Due to the more accurate description of the historical dependence on systems, various modeling methods and theories of fractional-order dynamic systems based on the definition of fractional calculus have gradually been successfully applied in many fields, such as high-energy physics, anomalous diffusion, complex viscoelastic material mechanics, system control, rheology, biomedical engineering, and economics [20-22]. Hereafter, several consensus problems are studied for fractional-order multi-agent systems (FOMASs) [23-28]. For example, in [23], the consensus of FOMASs with general linear models over a directed communication graph is 
investigated via an observer-type protocol. In [24], the leader-following tracking consensus has been studied in MASs in which the leader is described by an integer-order differential system while the followers are governed by a fractional-order differential system. In [25], sampled-data-based consensus problems for single-integrator FOMASs are studied. In [26], the authors investigate several consensus problems of continuous-time double-integrator FOMASs with directed communication topologies. In [27], the authors investigate the tracking consensus problem for second-order leader systems by designing a fractional-order observer, where a periodic sample-based data event-triggered control is employed. Very recently, [28] addresses the event-triggered consensus problem for Takagi-Sugeno fuzzy FOMASs with switching topologies.

In addition, it is well known that the real system is always in disturbance from internal fluctuation and the external environment. Therefore, it is undoubtedly of practical significance to study the stochastic dynamics of complex systems under noise disturbance. The consensus/synchronization under the action of noise is a typical dynamic characteristic of stochastic complex systems. Its occurrence mechanism and the influence of various factors on it need to be further studied. Several studies examined the consensus of MASs under noise disturbance [29-32]. In [29], a distributed sampled-data based protocol is proposed for the average consensus of second-order integral MASs under switching topologies and communication noises, and some sufficient conditions have been obtained to ensure the mean square/almost sure average consensus. The study in [30] studies the second-order consensus of MASs with noise in a leaderless architecture. Sufficient consensus conditions are established, which characterize how much the noise intensity or the delay MASs can stand so that second-order consensus can be reached. Ref. [31] investigates the consensus stabilization problem of stochastic MASs with noise, Markovian switching topology, and communication delays, and obtains necessary and sufficient conditions for the consensus stabilization. Subsequently, the authors in [32] investigate the consensus problem of multiple discrete-time integrator agents with communication constraints and additive process noise and propose a protocol to achieve the approximate consensus of agents over inter-agent communication networks with finite bit rates. However, there is little literature on the consensus of FOMASs under noise disturbance.

Moreover, the traditional view is that noise will inhibit the consensus/synchronization behavior of the system, but previous studies have shown that in many nonlinear systems, noise will affect the synchronization of the system in different forms, resulting in positive effects such as 'noise-induced synchronization' or 'noise-enhanced synchronization' [33]. Especially when the individuals of complex networks are in the same common environment, the environmental noise may have the same effect on them. For example, in the nervous system, the highly correlated and random synaptic inputs received by each neuron are usually modeled as the common noise of neurons [34]. Random fluctuations of the extracellular environment also have the same effect on each cell in the population because this noise exerts the same effect on each cell through the signal molecule AI $[35,36]$. Studies have shown that common extracellular noise can indeed make each cell in the population switch between two equilibrium states in cooperative behavior, so as to finally realize the body synchronous switching of the cell population [35]. In fact, as early as 1994, Maritan and Banavar found that two identical and independent chaotic maps affected by uniformly distributed common noise can be synchronized [37]. Subsequently, various positive effects of common noise in complex systems, such as promoting the synchronization of chaotic systems [38], inducing spatiotemporal coherent resonance [36], and stochastic resonance of integer-order or fractional-order complex networks $[39,40]$, etc., have attracted more and more attention. However, there is also little reported on the positive effect of noise on the consensus of MASs.

Motivated by the above discussion, in this paper, we present a double-integral FOMAS in a common bounded fluctuating potential where the protocol term consists of both the relative position and velocity information of neighboring agents. At first, based on the stability theory of fractional differential equations, the consensus conditions of the 
presented system in the absence of noise are analytically given and verified by a numerical simulation algorithm. From an analysis of the influences of fractional order and other system parameters (the eigenvalues of the Laplacian matrix, the double coupling strength, and the potential parameter) on the consensus of the presented double-integral FOMAS, it is found that the fractional-order system is easier to achieve position/velocity consensus than the classical integer-order system; that is, the fractional-order system has a larger range of consensus parameters. Then, the influences of the system order and other system parameters on the consensus of the presented system in the presence of bounded noise are also analyzed. It is found that common and bounded noise have a promoting effect on the consensus of double-integral FOMAS, while it does not promote the consensus of the corresponding integer-order MAS. That is to say, the bounded noise of appropriate intensity plays an optimization role for the presented FOMAS. To sum up, the research results of this paper, on the one hand, show that the fractional-order complex network under the action of noise has richer dynamic characteristics than the integer-order complex network. On the other hand, the presented FOMAS can be synchronized by changing the noise intensity. This method provided a brand-new way to realize consensus of fractional-order systems with uncontrollable system parameters by controlling the output noise intensity.

The rest of the paper is organized as follows. Section 2 firstly presents the new double-integral FOMAS in a bounded fluctuating potential, then deduces and verifies the consensus conditions, and analyzes the influences of the system order and other parameters on the consensus of the presented system in the absence of noise. In Section 3, the influences of the parameters on the consensus of the presented system in the presence of bounded noise are analyzed. Section 4 gives the conclusion of this paper.

\section{Model and Consensus Analysis of Double-Integral FOMAS}

\subsection{Double-Integral FOMAS Model and Its Consensus Conditions}

\subsubsection{Graph Theory}

Let $\mathcal{G}(\mathcal{A})=(\mathcal{V}, \mathcal{E}, \mathcal{A})$ denote an unweighted and undirected graph of order $N$, where $\mathcal{V}=\left\{v_{1}, v_{2}, \ldots, v_{N}\right\}$ is the vertex set of the graph, $\mathcal{E} \subset \mathcal{V} \times \mathcal{V}$ is the edge set, and $\mathcal{A}=\left(a_{i j}\right) \in \mathbb{R}^{N \times N}$ is an adjacency matrix with rows and columns indexed by the vertices. The edge of $G$ is denoted by a pair $\left(v_{i}, v_{j}\right)$, and $\left(v_{i}, v_{j}\right) \in \mathcal{E}$ if and only if $a_{i j}=1$. In this paper, we assume $\left(v_{i}, v_{i}\right) \notin \mathcal{E}$ and each element of $\mathcal{E}$ is unique and denote the collection of neighbors of $v_{i}$ as $\mathcal{N}_{i}=\left\{v_{j}:\left(v_{j}, v_{i}\right) \in \mathcal{E}\right\}$. We assume that $\mathcal{A}^{T}=\mathcal{A}$, that is, graph $\mathcal{G}(\mathcal{A})=(\mathcal{V}, \mathcal{E}, \mathcal{A})$ is undirected. Then the Laplacian matrix $L=\left(l_{i j}\right)$ corresponding to the coupling network can be derived as follows:

$$
l_{i j}=\left\{\begin{array}{l}
\sum_{v_{j} \in N_{i}} a_{i j}, \quad i=j \\
-a_{i j,} \quad i \neq j
\end{array}\right.
$$

Furthermore, for an undirected graph, the matrix $L$ is diagonalizable and has $N$ real eigenvalues such that $0=\lambda_{1} \leq \lambda_{2} \cdots \leq \lambda_{N}$.

\subsubsection{Double-Integral FOMAS}

We consider a FOMAS consisting of $N$ double linearly coupling agents, $x_{i}(t)$ represents the position of the $i$-th agent along the $x$ axis at time $t$, and $v_{i}(t)$ represents the velocity of the $i$-th agent along the $x$-axis at time $t . c$ and $d$ are the position coupling strength and velocity coupling strength, respectively. In addition, each agent is simultaneously affected by a random fluctuating potential field with a potential field parameter $a>0$, and the common noise of the system is $\xi(t)$. Thus, the model of the presented double-integral FOMAS can be expressed by the following fractional-order stochastic differential equations:

$$
\left\{\begin{array}{l}
D^{p} x_{i}(t)=v_{i}(t) \\
D^{p} v_{i}(t)=-(a+\xi(t)) x_{i}(t)-c \sum_{j=1}^{N} l_{i j} x_{j}(t)-d \sum_{j=1}^{N} l_{i j} v_{j}(t)
\end{array}, i=1,2, \ldots, N\right.
$$


where, $D^{p} x_{i}(t)$ and $D^{p} v_{i}(t)$ denote the $p$-th-order Caputo derivatives [20] of the position and velocity of the $i$-th agent, respectively. Here, we consider the case $p \in(0,1]$, when $p=1$, FOMAS (2) becomes the classical double-integral MAS.

Without the influence of noise, FOMAS (2) can be simplified to the following deterministic form:

$$
\left\{\begin{array}{l}
D^{p} x_{i}(t)=v_{i}(t) \\
D^{p} v_{i}(t)=-a x_{i}(t)-c \sum_{j=1}^{N} l_{i j} x_{j}(t)-d \sum_{j=1}^{N} l_{i j} v_{j}(t)
\end{array}, i=1,2, \ldots, N\right.
$$

With increase in time, all agents may converge asymptotically on the same consensus value. The definitions of every agent in above systems converging on the same consensus value are given as follows:

Definition 1. The noise-free FOMAS (3) is said to achieve consensus if for any initial states, $\lim _{t \rightarrow \infty}\left[x_{i}(t)-x_{j}(t)\right]=0$ and $\lim _{t \rightarrow \infty}\left[v_{i}(t)-v_{j}(t)\right]=0, \forall i, j=1,2, \ldots, N$. Furthermore, if there exist $x^{*}$, such that $\lim _{t \rightarrow \infty} x_{i}(t)=x^{*}$ and $\lim _{t \rightarrow \infty} v_{i}(t)=0, \forall i=1,2, \ldots, N$, then system (3) is said to achieve static consensus, where $\left[x^{*}, 0\right]^{T}$ is called as the consensus state of the system.

Definition 2. The stochastic FOMAS (2) is said to achieve consensus in the sense of mean, if for any initial states, $\lim _{t \rightarrow \infty}\left[\left\langle x_{i}(t)-x_{j}(t)\right\rangle\right]=0$ and $\lim _{t \rightarrow \infty}\left[\left\langle v_{i}(t)-v_{j}(t)\right\rangle\right]=0, \forall i, j=1,2, \ldots, N$, where $\langle$.$\rangle is in the sense of mathematical expectation. Furthermore, if there exist x^{*}$, such that $\lim _{t \rightarrow \infty}\left\langle x_{i}(t)\right\rangle=x^{*}$ and $\lim _{t \rightarrow \infty}\left\langle v_{i}(t)\right\rangle=0, \forall i=1,2, \ldots, N$, then system (2) is said to achieve static consensus, where $\left[x^{*}, 0\right]^{T}$ is called as the consensus state of the system.

\subsubsection{Consensus Conditions for Double-Integral FOMAS}

Firstly, we study the collective dynamic behavior of the presented double-integral FOMAS (2) - consensus of position/velocity in the absence of noise through theoretical analysis and derive the corresponding consensus conditions.

Equation (3) are differential equation systems of two sets of variables, which can be transformed into the following matrix form:

$$
D^{p} y_{i}=A_{0} y_{i}-\sum_{j=1}^{N} l_{i j} H y_{j}
$$

where, $y_{i}=\left[\begin{array}{l}x_{i} \\ v_{i}\end{array}\right], A_{0}=\left[\begin{array}{cc}0 & 1 \\ -a & 0\end{array}\right], H=\left[\begin{array}{ll}0 & 0 \\ c & d\end{array}\right]$

Obviously, Equation (4) has a unique zero-solution synchronization state $\bar{y}(t)=0$. Let $\delta_{i}=y_{i}-\bar{y}, \delta=\left[\delta_{1}, \delta_{2}, \cdots \delta_{N}\right]$, the variational equation near the synchronization state corresponding to Equation (3) can be obtained as follows:

$$
D^{p} \delta=A_{0} \delta-H \delta L^{\top}
$$

Because the Laplacian matrix $L$ of Equation (4) can be diagonalized, $L$ can be decomposed as $L=P \Lambda P^{-1}$, where the diagonal matrix $\Lambda=\operatorname{diag}\left(\lambda_{1}, \lambda_{2}, \cdots, \lambda_{N}\right), \lambda_{k}$ are the eigenvalues of the Laplacian matrix $L$ and satisfies $0=\lambda_{1} \leq \lambda_{2} \cdots \leq \lambda_{N}$. Multiply both sides of Equation (4) by $P$, and let $\eta=\delta P=\left[\eta_{1}, \ldots, \eta_{N}\right]$, then we can obtain the following equation of $\eta$ :

$$
D^{p} \boldsymbol{\eta}=A_{0} \boldsymbol{\eta}-H \boldsymbol{\eta} \Lambda
$$

The above Equation (6) can be expressed as follows:

$$
D^{p} \eta_{k}=\left(A_{0}-\lambda_{k} H\right) \eta_{k}=B_{k} \eta_{k}, k=2, \ldots, N
$$

where $B_{k}=A_{0}-\lambda_{k} H=\left[\begin{array}{cc}0 & 1 \\ -a-c \lambda_{k} & -d \lambda_{k}\end{array}\right]$. 
According to [41], for $k=1$ we have the variational equation for the synchronization manifold $\bar{y}(t)=0\left(\lambda_{1}=0\right)$. All other equations with $k \geq 2$ in Equation (7) correspond to transverse eigenvectors and represent dynamics perpendicular to the direction (transverse) of the modified manifold, also called the transverse system. So, we have succeeded in separating the synchronization manifold from the other transverse directions. The convergence of the latter indicates that the system is tending to synchronization state. Thus, if all the equations with $k \geq 2$ in (7) correspond to the transverse system being stable, then the complete consensus/synchronization of the system can occur and is stable.

Thus, the transverse system of Equation (7) can be written as the fractional-order main stability equation $D^{p} \mathbf{y}=B_{k} \mathbf{y}, k \geq 2$. According to the stability theory of the zero solution of fractional differential equations [20], the global asymptotic stability of its zero solution is equivalent to the argument of all eigenvalues of $B_{k}$ satisfies $|\arg (\lambda)|>p \pi / 2$. That is to say, when the arguments of all eigenvalues $\lambda$ of $B_{k}(k=2,3, \ldots, N)$ satisfy $|\arg (\lambda)|>p \pi / 2$, the noise-free double-integral FOMAS (3) achieves consensus given in Definition 1.

After theoretical derivation, the following theorem gives the consensus conditions of the presented double-integral FOMAS (3) when the three parameters-the potential field parameter $a>0$, the position coupling strength $c$ and the velocity coupling strength $d$ take different types of values.

Theorem 1. Consider the noise-free double-integral FOMAS (3) under an unweighted and undirected graph with fractal order $p \in(0,1]$, the potential field parameter $a>0$, the position coupling strength $c \in R$, the velocity coupling strength $d \in R$. The consensus in Definition 1 can be achieved if and only if $a, c, d$, and the largest eigenvalue $\lambda_{\text {max }}$ of the Laplacian matrix $L$ satisfy one of the following three categories:

(1) Category 1: $d \geq 0, c \geq 0$.

(2) Category 2: $d \geq 0, c<0$, and the largest eigenvalue of the Laplacian matrix L satisfies $0<\lambda_{\max }<-a / c$.

(3) Category 3: $d<0$, and the largest eigenvalue of the Laplacian matrix L satisfies

$$
0<\lambda_{\max }<d^{-2} \cos ^{2}(p \pi / 2)\left[2 c+2 \sqrt{c^{2}+a d^{2} \sec ^{2}(p \pi / 2)}\right]
$$

In particular, this category, when $p=1$, the corresponding integer-order MAS cannot achieve consensus.

Proof. The consensus conditions of the noise-free FOMAS (3) depends on the eigenvalues of the matrix $B_{k}$ in the fractional main stability equation $D^{p} \mathbf{y}=B_{k} \mathbf{y}, k \geq 2$. Since the characteristic equation of matrix $B_{k}$ can be written as $\operatorname{det}(B-\lambda E)=\lambda^{2}+\left(d \lambda_{k}\right) \lambda+(a+$ $\left.c \lambda_{k}\right)=0$, whose arguments of all the eigenvalues can be calculated. Here, since $\lambda_{k}$ is an eigenvalue of the Laplacian matrix $L, \lambda_{k}$ needs to satisfy $\lambda_{k} \geq 0$. When $\lambda_{k}=0$, Equation (7) can be written as $D^{\alpha} \eta_{k}=A \eta_{k}$, so the characteristic polynomial of matrix $B_{1}$ in the fractionalorder main stability equation is $\operatorname{det}\left(B_{1}-\lambda E\right)=\lambda^{2}+a$, let $\lambda^{2}+a=0$, and the eigenvalue of $B_{1}$ can be calculated as $\lambda= \pm i \sqrt{a}$, which leads to the stability of the corresponding main stability equation. When $\lambda_{k} \neq 0$, the roots of the matrix characteristic equation depend on the value of its discriminant $\Delta=\left(d \lambda_{k}\right)^{2}-4\left(a+c \lambda_{k}\right)$, as follows:

(1) When $\Delta>0$, that is $\lambda_{k}>d^{-2}\left(2 c+2 \sqrt{c^{2}+a d^{2}}\right)$ or $\lambda_{k}<d^{-2}\left(2 c-2 \sqrt{c^{2}+a d^{2}}\right)$, then $B_{k}$ has two different eigenvalues $\lambda_{B}^{1,2}=\left[-d \lambda_{k} \pm \sqrt{\left(d \lambda_{k}\right)^{2}-4\left(a+c \lambda_{k}\right)}\right] / 2$;

(2) When $\Delta=0$, that is $\lambda_{k}=d^{-2}\left(2 c+2 \sqrt{c^{2}+a d^{2}}\right)$ or $\lambda_{k}=d^{-2}\left(2 c-2 \sqrt{c^{2}+a d^{2}}\right)$, then $B_{k}$ has two identical eigenvalues $\lambda_{B}^{1,2}=-d \lambda_{k} / 2$.

(3) When $\Delta<0$, that is $d^{-2}\left(2 c-2 \sqrt{c^{2}+a d^{2}}\right)<\lambda_{k}<d^{-2}\left(2 c+2 \sqrt{c^{2}+a d^{2}}\right)$, then $B_{k}$ has two different eigenvalues $\lambda_{B}^{1,2}=\left[-d \lambda_{k} \pm i \sqrt{4\left(a+c \lambda_{k}\right)-\left(d \lambda_{k}\right)^{2}}\right] / 2$. 
Now that we discuss the eigenvalue argument value of $B_{k}$ in the following five cases:

Case 1: $(d>0, c \geq 0)$ : No matter $\Delta=\left(d \lambda_{k}\right)^{2}-4\left(a+c \lambda_{k}\right)$ is any real number, we have that the real part of all eigenvalues of $B_{k}$ is less than 0 (especially, when $\Delta>0$, since $-d \lambda_{k}<$ $-\sqrt{\left(d \lambda_{k}\right)^{2}-4\left(a+c \lambda_{k}\right)}$ then we have $\left.\lambda_{B}^{1,2}=\left[-d \lambda_{k} \pm \sqrt{\left(d \lambda_{k}\right)^{2}-4\left(a+c \lambda_{k}\right)}\right] / 2<0\right)$. Thus, all the eigenvalue arguments of $B_{k}$ satisfy $|\arg (\lambda)|>p \pi / 2$. So, in this case, with $\lambda_{k}$ being any positive real numbers, the noise-free FOMAS (3) can achieve consensus.

Case 2: $(d>0, c<0)$ : When $\Delta=\left(d \lambda_{k}\right)^{2}-4\left(a+c \lambda_{k}\right)>0$, that is to say $\lambda_{k}<$ $d^{-2}\left(2 c-2 \sqrt{c^{2}+a d^{2}}\right)$ or $d^{-2}\left(2 c+2 \sqrt{c^{2}+a d^{2}}\right)<\lambda_{k}, B_{k}$ has two different real eigenvalues $\lambda_{B}^{1,2}=\left[-d \lambda_{k} \pm \sqrt{\left(d \lambda_{k}\right)^{2}-4\left(a+c \lambda_{k}\right)}\right] / 2$, then both eigenvalues $\lambda_{B}^{1,2}$ are less than 0 if and only if $\lambda_{k}<-a / c$. Note that in this case $d^{-2}\left(2 c+2 \sqrt{c^{2}+a d^{2}}\right)<-a / c$, we obtain the condition of $\lambda_{k}$ is $\lambda_{k}<-a / c$; when $\Delta=0$ or $\Delta<0$, it is easy to find that the real part of all eigenvalues of $B_{k}$ is less than 0 , thus all the eigenvalue arguments of $B_{k}$ satisfy $|\arg (\lambda)|>p \pi / 2$. Therefore, in this case, when each $\lambda_{k}$ satisfies $\lambda_{k}<-a / c$, the noise-free FOMAS (3) can achieve consensus.

Case 3: $(d=0, c \geq 0)$ : It is easy to find that the discriminant always satisfies $\Delta=$ $-4\left(a+c \lambda_{k}\right)<0$, then the eigenvalues of $B_{k}$ are pure imaginary numbers $\lambda_{B}^{1,2}= \pm i \sqrt{a+c \lambda_{k}}$ which satisfy $|\arg (\lambda)|>p \pi / 2$, in this case the noise-free FOMAS (3) can always achieve consensus.

Case 4: $(d=0, c<0)$ : When $\Delta=-4\left(a+c \lambda_{k}\right) \geq 0$, that is $\lambda_{k} \geq-a / c$, the eigenvalues of $B_{k}$ are $\lambda_{B}^{1,2}= \pm \sqrt{-\left(a+c \lambda_{k}\right)}$, in this case, the arguments of the eigenvalues of $B_{k}$ do not all satisfy $|\arg (\lambda)|>p \pi / 2$, so the noise-free fractional-order system (2) cannot achieve consensus. However, when $\Delta<0$, that is $\lambda_{k}<-a / c$, the eigenvalue of $B_{k}$ are pure imaginary numbers $\lambda_{B}^{1,2}= \pm i \sqrt{a+c \lambda_{k}}$, which satisfy $|\arg (\lambda)|>p \pi / 2$. Thus, in this case, the noise-free FOMAS (3) can achieve consensus if each $\lambda_{k}<-a / c$.

Case 5: $(d<0)$ : when $\Delta \geq 0$, the eigenvalue $\lambda_{B}^{1,2}=\left[-d \lambda_{k} \pm \sqrt{\left(d \lambda_{k}\right)^{2}-4\left(a+c \lambda_{k}\right)}\right] / 2$ of $B_{k}$ are always positive real numbers, the arguments of the eigenvalues of $B_{k}$ do not all satisfy $|\arg (\lambda)|>p \pi / 2$, so the noise-free FOMAS (3) cannot achieve consensus. However, when $\Delta<0$, the eigenvalues of $B_{k}$ are $\lambda_{B}^{1,2}=\left[-d \lambda_{k} \pm i \sqrt{4\left(a+c \lambda_{k}\right)-\left(d \lambda_{k}\right)^{2}}\right] / 2$, and their real parts are always positive. At this time, the arguments of the eigenvalues of $B_{k}$ satisfy $|\arg (\lambda)|>p \pi / 2$ if and only if the argument of the eigenvalue satisfies the following:

$$
\tan (|\arg (\lambda)|)=-\sqrt{4\left(a+c \lambda_{k}\right)-\left(d \lambda_{k}\right)^{2}} /\left(d \lambda_{k}\right)>\tan (p \pi / 2)
$$

Note that $d^{-2} \cos ^{2}\left(\frac{p \pi}{2}\right)\left[2 c-2 \sqrt{c^{2}+a d^{2} \sec ^{2}\left(\frac{p \pi}{2}\right)}\right]<0$. Therefore, Equation (8) can be solved as follows:

$$
0<\lambda_{\text {max }}<d^{-2} \cos ^{2}\left(\frac{p \pi}{2}\right)\left[2 c+2 \sqrt{c^{2}+a d^{2} \sec ^{2}\left(\frac{p \pi}{2}\right)}\right]
$$

So, in this case, when $\lambda_{k}$ satisfies Equation (9), the system can achieve consensus.

\subsection{Simulation Verification of Consensus Conditions}

In Section 2.1.3, we theoretically derive the consensus conditions for the noise-free FOMAS (3) under different parameter situations. In this subsection, we verify the above theoretical consensus conditions through numerical simulations.

Let $x_{i}\left(t_{k}\right)$ and $v_{i}\left(t_{k}\right)$ represent the position and velocity of the $i$-th agent at time $t=k h$, respectively, and $h$ is the iterative step size. Based on the finite difference method defined 
by Grunwald-Letnikow [20], the numerical iterative formula of the double-integral FOMAS (2) is given as follows:

$$
\left\{\begin{array}{l}
x_{i}\left(t_{k}\right)=v_{i}\left(t_{k}\right) h^{p}-\sum_{r=1}^{k-1}(-1)^{r}\left(\begin{array}{c}
p \\
r
\end{array}\right) x_{i}\left(t_{k-r}\right) \\
v_{i}\left(t_{k}\right)=\left[-\left(a+\xi\left(t_{k}\right)\right) x_{i}\left(t_{k}\right)-c \sum_{j=1}^{N} l_{i j} x_{j}\left(t_{k}\right)-d \sum_{j=1}^{N} l_{i j} v_{j}\left(t_{k}\right)\right] h^{p}-\sum_{r=1}^{k-1}(-1)^{r}\left(\begin{array}{c}
p \\
r
\end{array}\right) v_{i}\left(t_{k-r}\right)
\end{array} .\right.
$$

Now we verify the consensus conditions (Theorem 1 given in Section 2.1.2) of the double-integral FOMAS (2) in three categories ((1) $d \geq 0 ; c \geq 0$; (2) $d \geq 0, c<0$; (3) $d<0)$. In order to quantitatively describe the tendency of the system consensus changing with time, we introduce the following system average position difference function:

$$
x_{\text {diff }}(t)=\frac{1}{N-1} \sum_{n=1}^{N-1}\left|\left\langle x_{n}(t)-x_{n+1}(t)\right\rangle\right|
$$

Additionally, the system average velocity difference function as follows:

$$
v_{\text {diff }}(t)=\frac{1}{N-1} \sum_{n=1}^{N-1}\left|\left\langle v_{n}(t)-v_{n+1}(t)\right\rangle\right|
$$

to measure the consensus of the position and velocity of all the agents, respectively.

The number of the agents in the simulations of this paper is set to $N=10$, the coupling network is generated using a Watts-Strogatz (WS) small-world network rule, with average degree $K_{c}=4$ and reconnection probability $p_{c}=0.5$. The maximum eigenvalue of the corresponding Laplacian matrix $L$ is $\lambda_{\max }=7.3738$. Meanwhile, the simulation step size is set to $h=0.005$, and the simulation duration is $T=30 \pi$.

(1) When the position coupling strength $c=1$, the velocity coupling strength $d=0.1$, and the potential field parameter $a=1$, Figure 1 shows the time-varying curves of the average position difference function $x_{\text {diff }}(t)$ and average velocity difference function $v_{\text {diff }}(t)$ in Equations (11) and (12) of the FOMAS (3) under different orders. It can be seen that for all fractional orders, the values of the average position difference function $x_{\text {diff }}(t)$ and the average velocity difference function $v_{\text {diff }}(t)$ gradually decrease as time increases, and converge to a minimum value, indicating that all agents tend to be consistent in terms of position and velocity. In addition, it can be seen that the larger the order, the lower the value of the average position difference function and the average velocity difference function at the same time, indicating that the system achieves synchronization faster.
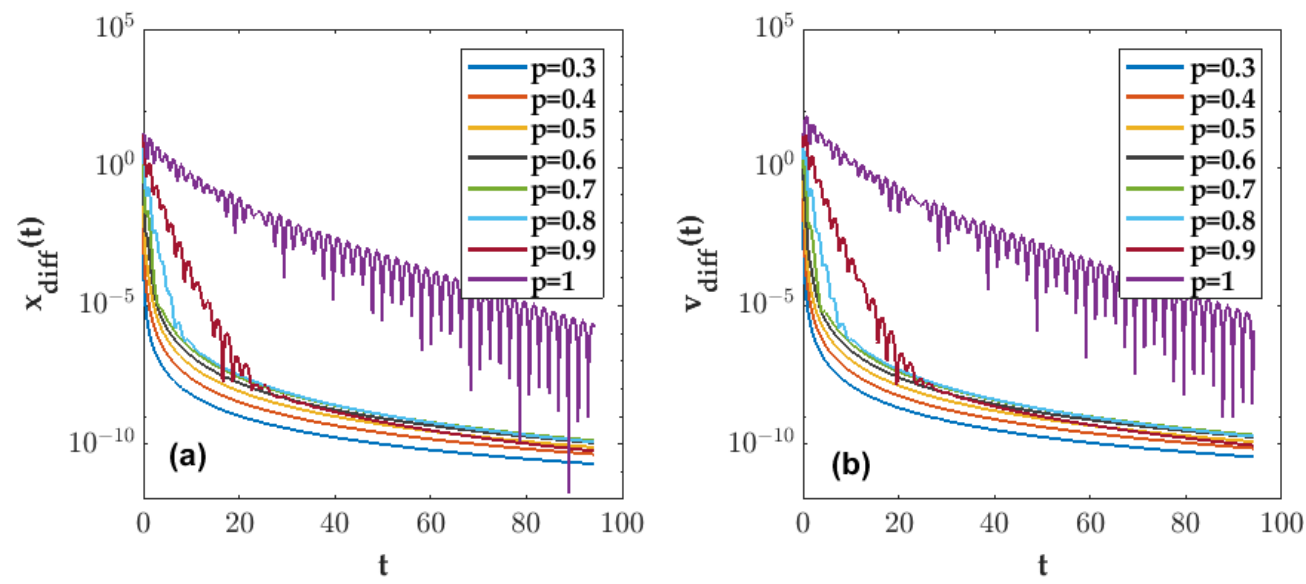

Figure 1. Average position difference function $x_{\text {diff }}(t)(a)$ and average velocity difference function $v_{\text {diff }}(t)(\mathbf{b})$ curves of time of the FOMAS (3) with different orders $p$ (the case $d \geq 0, c \geq 0$ ). 
Under the same simulation parameters in Figure 1, Figure 2 plots the time-varying curves of the position and velocity of each agent when the fractional order $p=0.8$. It can be found that the starting values of velocity and position of each agent are different, but they gradually tend to the average value of zero before $10 \mathrm{~s}$, and then fluctuate around the average and finally converge to the same value of zero.
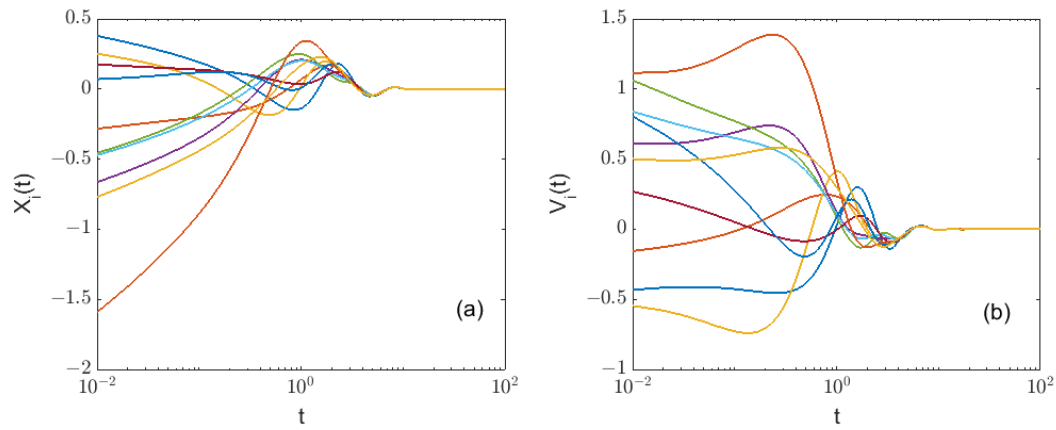

Figure 2. The curves of the position (a)/velocity (b) of each agent as a function of time of FOMAS (3) with different orders $p$ (the case $d \geq 0, c \geq 0$ ).

(2) When the position coupling strength $c=-1$ and the velocity coupling strength $d=0.1$, Figure $3 a-d$ respectively show the curves of the average position/velocity difference functions of the FOMAS (3) changing with time under different orders. In Figure $3 \mathrm{a}, \mathrm{b}, a=1$, and thus $1=-a / c<\lambda_{\max }=7.3738$, which does not satisfy the consensus conditions in Theorem 1, it can be found that all the curves show a trend of rapid growth to infinity as time increases. In Figure $3 c, d, a=20$, and thus $20=-a / c>\lambda_{\max }=7.3738$, which satisfies the consensus conditions in Theorem 1, compared with Figure $3 \mathrm{a}, \mathrm{b}$, all curves in Figure $3 \mathrm{c}, \mathrm{d}$ tend to zero as time increases. At the same time, it can be seen that the larger the order, the lower the average position/velocity difference functions at the same time, and the faster the system achieves consensus.
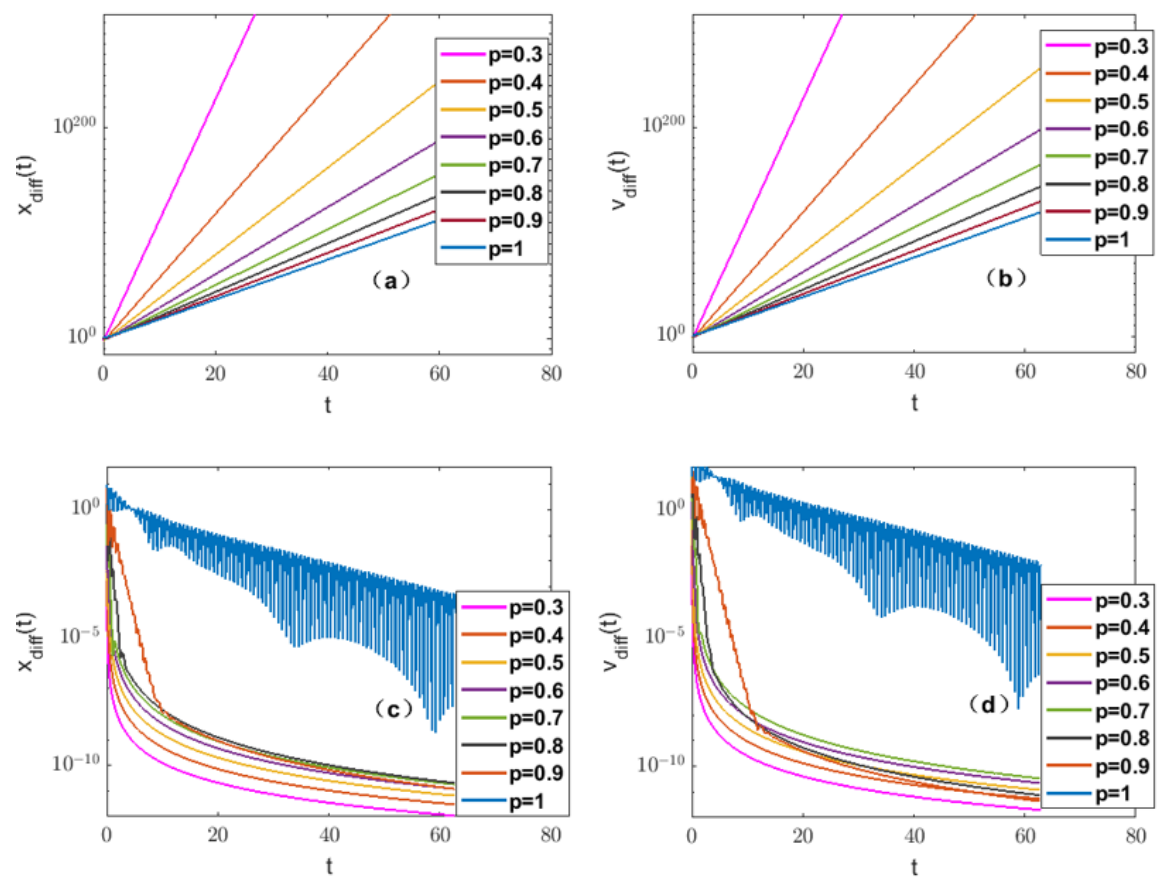

Figure 3. Average position difference function $x_{\text {diff }}(t)(\mathbf{a}, \mathbf{c})$ and average velocity difference function $v_{\text {diff }}(t)(\mathbf{b}, \mathbf{d})$ curves of time of the fractional-order multi-agent system (3) with different orders $p$ when $a=1(\mathbf{a}, \mathbf{b})$ and $a=20(\mathbf{c}, \mathbf{d})$ (the case $d \geq 0, c<0)$. 
Under the same simulation conditions as Figure 3, Figure 4 plots the time-varying curves of the position and velocity of each agent as a function of time when the order is $p=0.8$, where the potential field parameter in Figure $4 \mathrm{a}, \mathrm{b}$ is $a=1$, and in Figure $4 \mathrm{c}, \mathrm{d}$ is $a=20$. It can be found that when the parameters do not meet the consensus conditions of Category (2) in Theorem 1, the position and velocity of each agent diverge to farther values in a relatively short period of time, and there is a tendency to continue to diverge. When the parameters meet the consensus conditions of Category (2), the position and velocity of each agent fluctuates from different initial values tend to the average value of zero with the fluctuation amplitude gradually decaying, and finally achieve consensus.
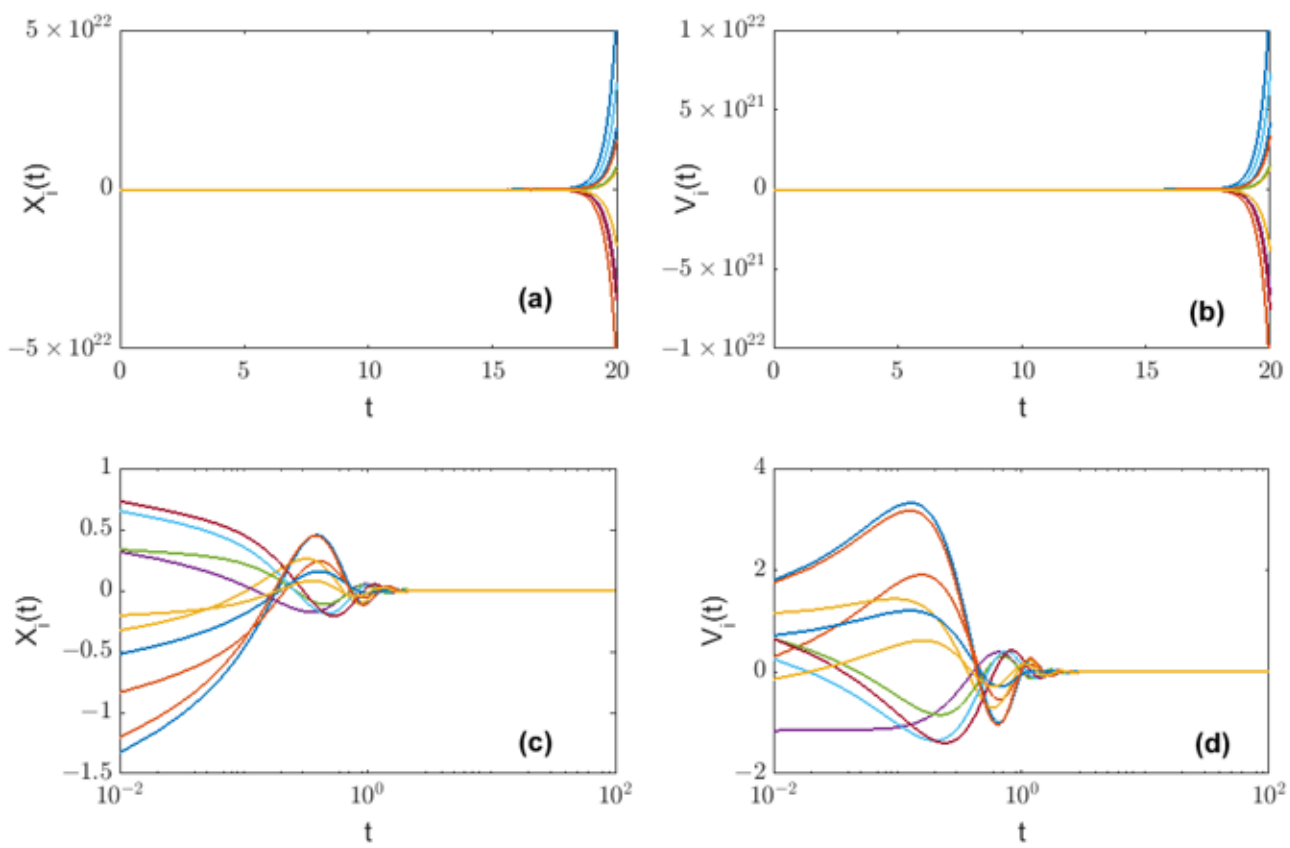

Figure 4. The curves of the position $(\mathbf{a}, \mathbf{c}) /$ velocity $(\mathbf{b}, \mathbf{d})$ of each agent as a function of time of FOMAS (3) with different orders $p$ when $a=1(\mathbf{a}, \mathbf{b})$ and $a=20(\mathbf{c}, \mathbf{d})$ (the case $d \geq 0, c<0$ ).

When the position coupling strength $c=0.1$, the velocity coupling strength $d=-0.1$, and the potential field parameter $a=0.4$, Figure 5 shows the curve of the average position/velocity difference function of each agent changing with time under different orders. According to the Category (3) in Theorem 1, FOMAS (3) may not always achieve consensus with different fractional orders $p$. When the order $p_{0}$ satisfies

$$
\lambda-2 \cos ^{2}\left(p_{0} \pi / 2\right)\left[2 c+2 \sqrt{c^{2}+a d^{2} \sec ^{2}\left(p_{0} \pi / 2\right)}\right]_{\max }
$$

are called as the critical consensus order. That is, when the order $p<p_{0}$, the FOMAS (3) achieves consensus. The critical consensus order under the current simulation is $p_{0}=0.7753$. It can be seen that when the order $p$ is greater than the critical consensus order $p_{0}$ (for example, $p_{0}=0.8,0.9,1$ ), the average position/velocity difference functions diverge to infinity, and the larger the order, the faster the divergence speed. When the order is smaller than the system critical consensus order, both the average position difference function and the average velocity difference function converge to zero, and the smaller the order, the faster the convergence speed. 

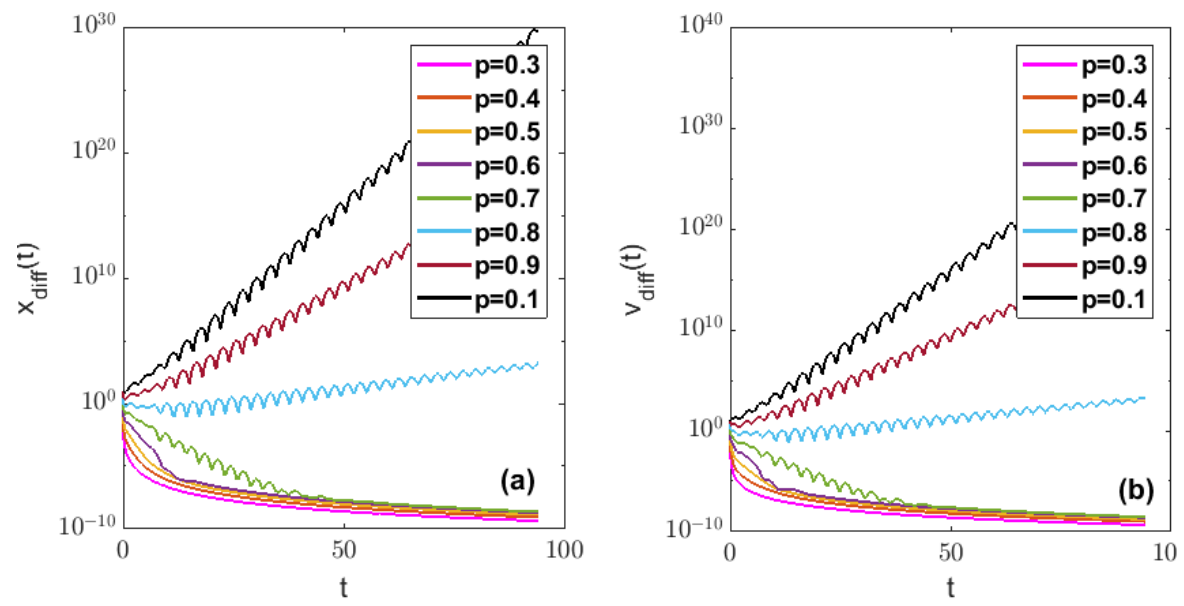

Figure 5. Average position difference function $x_{\text {diff }}(t)(\mathbf{a})$ and average velocity difference function $v_{\text {diff }}(t)(\mathbf{b})$ curves of time of the double-integral FOMAS (3) with different orders $p$ (the case $d<0$ ).

Under the same simulation conditions as Figure 5, Figure 6 plots the time-varying curves of position and velocity for each agent when the fractional order $p=0.8$ Figure $6 \mathrm{a}, \mathrm{b}$ and $p=0.3$ Figure $6 \mathrm{c}, \mathrm{d}$, respectively. It can be found that when the value of the fractional order does not meet the consensus condition of Category (3) $(p=0.8)$, the position and velocity of each agent show quasi-sinusoidal fluctuations with larger and larger amplitudes around 0 as time increases. However, when the order value satisfies the consensus condition of Category (3) $(p=0.3)$, the position and velocity of each agent gradually converge from different initial values to near the average value of zero.

All the above simulation results verify the consensus conditions for the noise-free FOMAS (3) given in Theorem 1.

\subsection{Influence of Parameters on the Consensus of the Double-Integral FOMAS}

In this section, we discuss the influence of position coupling strength $c$, velocity coupling strength $d$, and the potential field parameter $a$ on the consensus of the noise-free FOMAS (3) under different orders. In order to quantitatively characterize the trend of consensus with various parameters, we introduce the following average synchronization error between the positions and velocities of the multi-agent:

$$
\operatorname{Syn}_{x}=\left\langle\lim _{T \rightarrow+\infty} \int_{0}^{T} \frac{1}{N} \sum_{i=1}^{N}\left|x_{i}(t)-\bar{x}(t)\right| d t\right\rangle, \operatorname{Syn}_{v}=\left\langle\lim _{T \rightarrow+\infty} \int_{0}^{T} \frac{1}{N} \sum_{i=1}^{N}\left|v_{i}(t)-\bar{v}(t)\right| d t\right\rangle
$$

to describe the degree of consensus of the position and velocity of multiple agents, respectively. Here, $\bar{x}(t)=\sum_{i=1}^{N} x_{i}(t)$ and $\bar{v}(t)=\sum_{i=1}^{N} v_{i}(t)$ are the mean filed function of position and velocity, respectively. The smaller the value of $S y n_{x}$ and $S y n_{v}$, the stronger the consensus degree of the agents. Among them, $\langle\cdot\rangle$ represent the expectation operation, which is the result of one simulation in the absence of noise, and the average result of multiple simulations in the presence of noise. In the following simulations, the coupling network still uses the W-S small word network with 10 agents given in Section 2.2. 

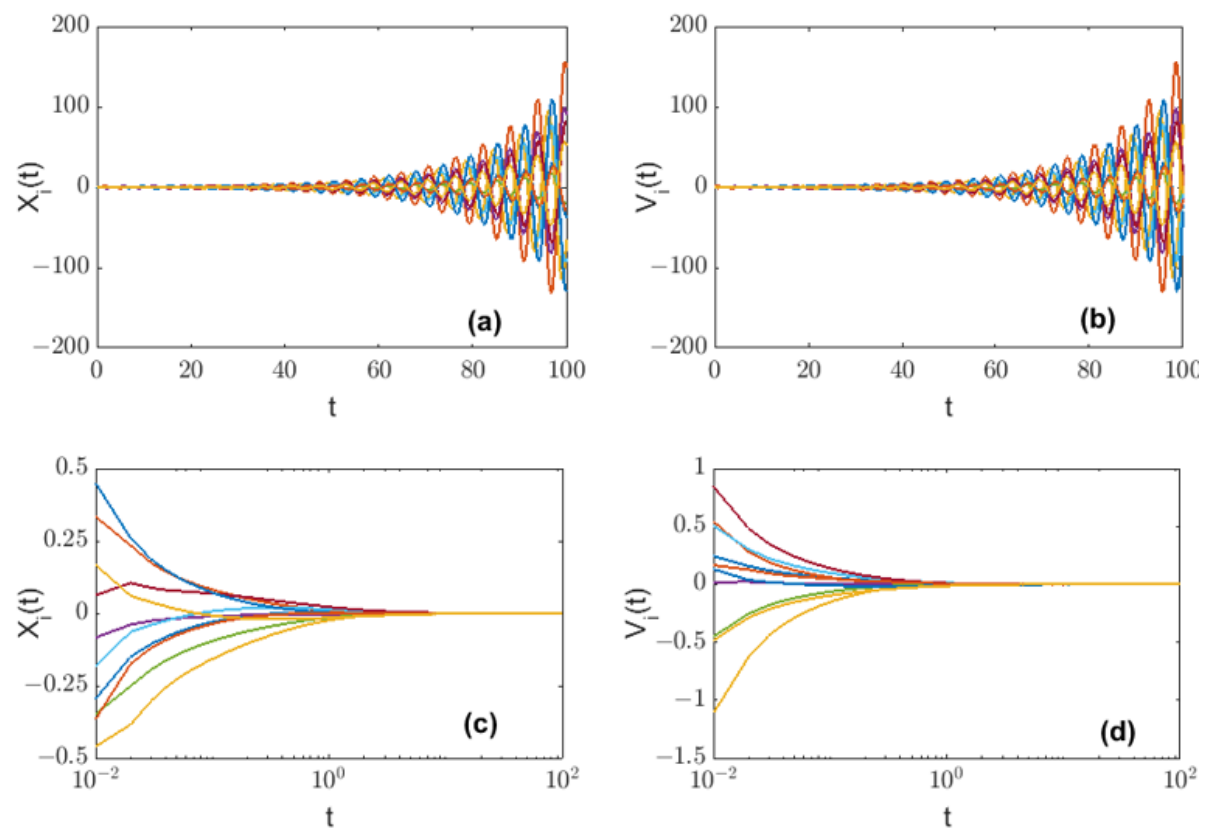

Figure 6. The curves of the position/velocity of each agent as a function of time of FOMAS (3) with orders $p=0.8(\mathbf{a}, \mathbf{b})$ and $p=0.3(\mathbf{c}, \mathbf{d})$ (the case $d<0)$.

\section{(1) Influence of potential parameter $a$}

When the position coupling strength $c=1$, the velocity coupling strength $d=-0.1$, Figure 7 shows the curves of the average synchronization error $S y n_{x}$ and $S y n_{v}$ as functions of the potential parameter $a$ under different orders $p=0.5,0.7,0.9,1$, respectively. It can be seen that the average synchronization errors $S y n_{x}$ and $S y n_{v}$ corresponding to the three curves of fractional order $p=0.5,0.7,0.9$ both decrease as the potential parameter $a$ increases, but the curves with integer order $p=1$ increases with the increase in the potential parameter $a$ and tends to infinity, which indicates that the influence of the potential parameter $a$ on the system consensus has completely different performances in the integerorder system and fractional-order multi-agent systems.
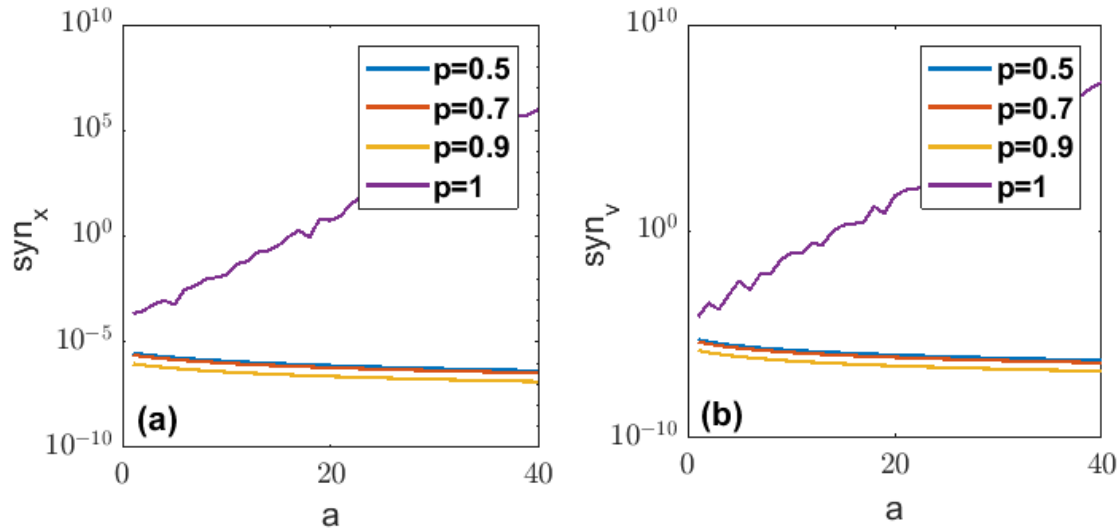

Figure 7. The average synchronization error $S y n_{x}(\mathbf{a})$ and $S y n_{v}(\mathbf{b})$ as functions of the potential parameter $a$ with different orders $p=0.5,0.7,0.9,1$.

(2) Influence of position coupling strength $c$

When the potential parameter $a=1$, the velocity coupling strength $d=-0.1$, Figure 8 shows the curves of the average synchronization error $S y n_{x}$ and $S y n_{v}$ as functions of the position coupling strength $c$ under different orders $p=0.5,0.7,0.9,1$, respectively. It can be seen that, as the position coupling strength $c$ changes from -0.5 to 2 , average 
synchronization error $S y n_{x}$ and $S y n_{v}$ generally show a downward trend, but there is an obvious turning point at $c=0$, indicating that the influence of the position coupling strength $c$ greater than 0 and less than 0 on the consensus is completely different. In addition, compared with the fractional smooth descent, when the order $p=1$, the average synchronization error tends to zero more slowly and has less fluctuation.
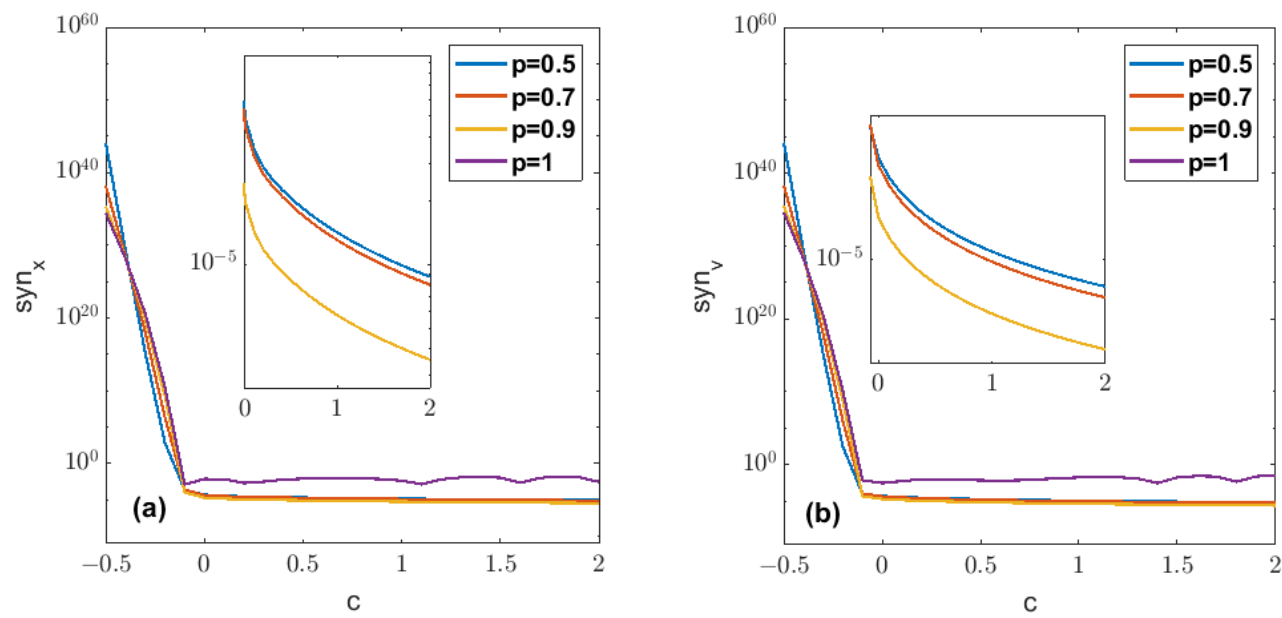

Figure 8. The average synchronization error $\operatorname{Syn}_{x}(\mathbf{a})$ and $S y n_{v}(\mathbf{b})$ as functions of the position coupling strength $c$ with different orders $p=0.5,0.7,0.9,1$.

(3) Influence of velocity coupling strength $d$

When the potential parameter $a=1$, the position coupling strength $c=1$, Figure 9 shows the curves of the average synchronization error $S y n_{x}$ and $S y n_{v}$ as functions of the velocity coupling strength $d$ under different orders $p=0.5,0.7,0.9,1$, respectively. It can be seen that as the velocity coupling strength gradually increase from -1 to 1 , the average synchronization error $S y n_{x}$ and $S y n_{v}$ begin to diverge to infinity, and as $d$ increases, different orders start to converge to 0 at different critical points of $d$. In addition, after crossing the critical point, the average synchronization error function first decreased and then increased, which is a nonlinear function of $d$. That is to say, as $d$ continued to increase, the synchronization error function began to decrease, and there was an optimal value of $d$ near the critical point to minimize the average synchronization error functions.
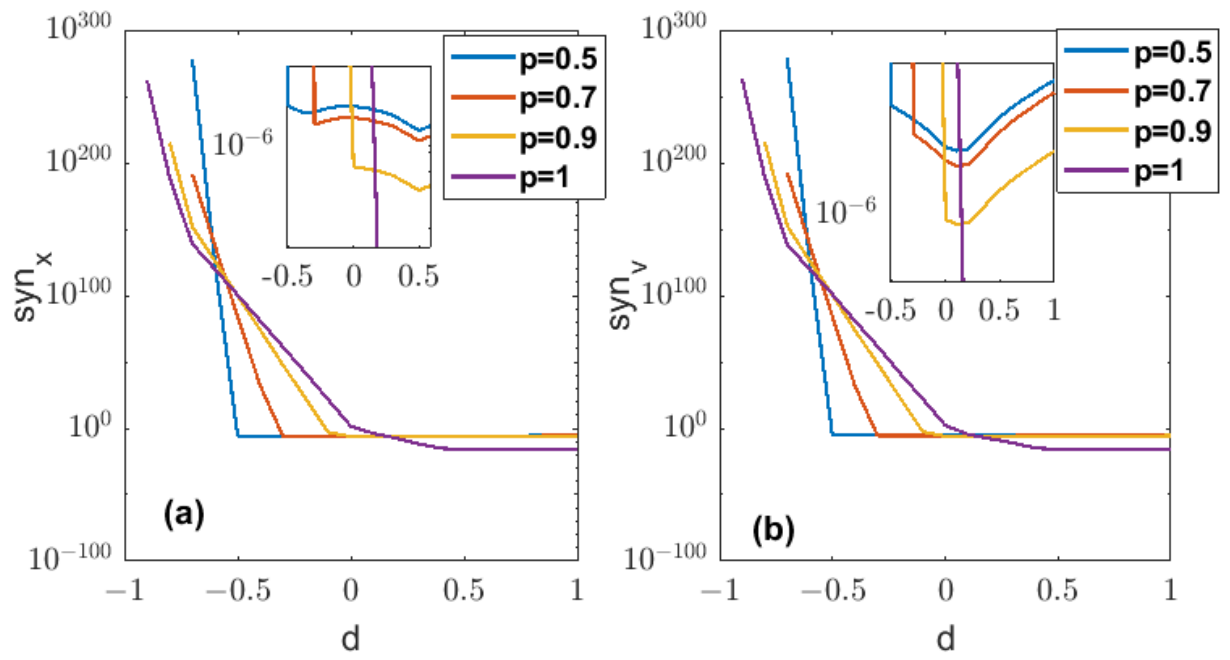

Figure 9. The average synchronization error $\operatorname{Syn}_{x}(\mathbf{a})$ and $S y n_{v}(\mathbf{b})$ as functions of the velocity coupling strength $d$ with different orders $p=0.5,0.7,0.9,1$. 


\section{Consensus Analysis of Double-Integral FOMAS in a Bounded Fluctuating Potential}

In this section, we analyze the synchronization dynamics of the presented FOMAS (2) under the disturbance of different types of random noise and the influence of noise on system consensus through the numerical simulation algorithm given by Equation (10). Among them, Section 3.1 sets the common noise $\xi(t)$ in Equation (2) as the following two types of fully bounded noise: a uniform noise (as shown in Figure 10a) and a Gaussian noise controlled by a cosine function (as shown in Figure 10b), respectively, Section 3.2 set $\xi(t)$ as a Gaussian noise that is not completely bounded (as shown in Figure 10c).
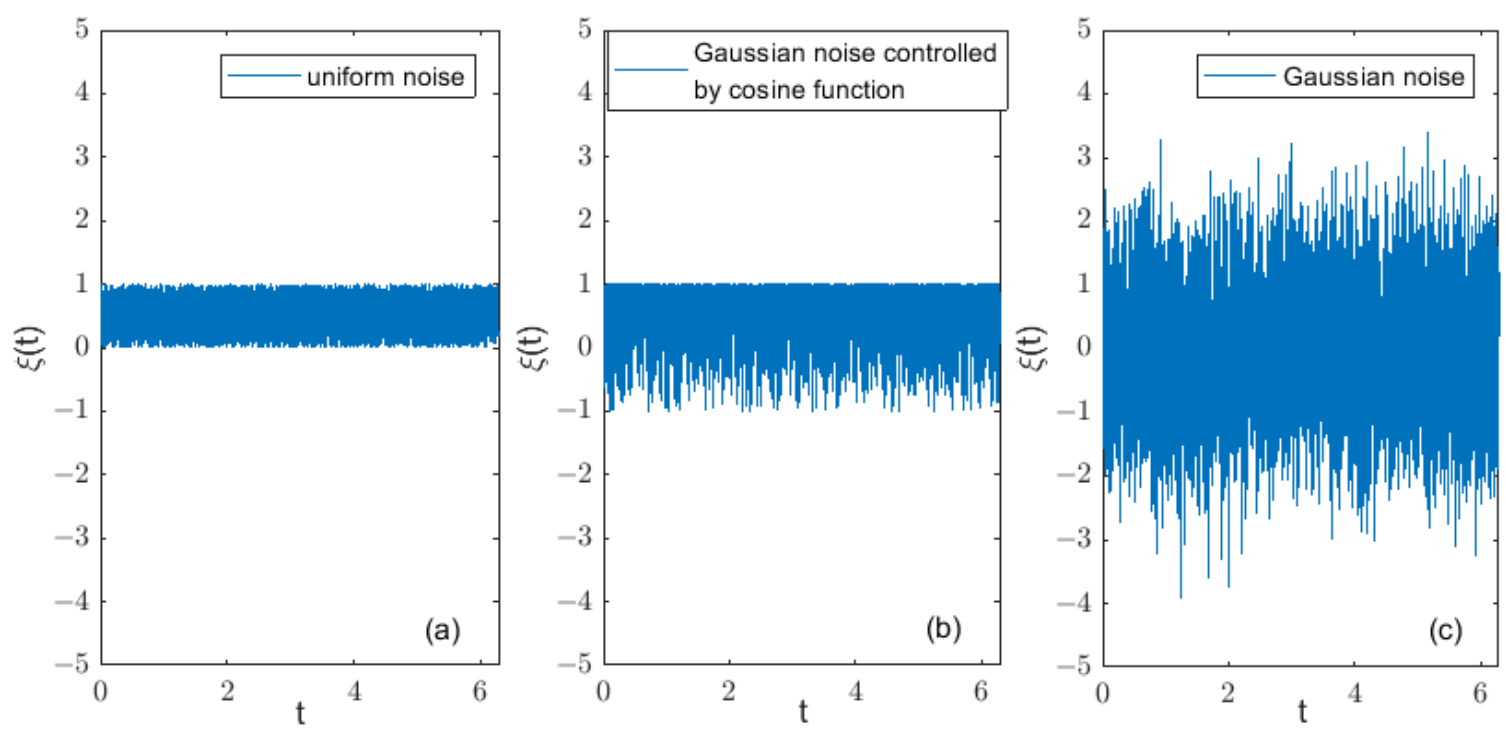

Figure 10. Uniform noise (a), Gaussian noise controlled by cosine function (b) and Gaussian noise (c) with intensity of 1.

\subsection{The Influence of Bounded Noise on System Consensus}

Considering the synchronization dynamics of FOMAS (2) when the common noise $\xi(t)$ is a fully bounded noise, we first analyze the influence of fractional-order on system consistency. When $\xi(t)$ is a bounded uniform noise, Figure 11 shows the average position synchronization error $S y n_{x}$ and the average velocity synchronization error $S y n_{v}$ curves of the noise intensity $D$ of the FOMAS (2) with different fractional-orders $p$. The coupling network is still the network with 10 agents given in Section 2.1, and other parameters are set as $a=1, c=0.1, d=0.1$. We can see that, for the classical integer-order system with $p=1$, both the average synchronization errors of both position and velocity will increase and tend to infinity with the increase in noise intensity $D$. However, for the fractional system with order $p<$ one, the average position synchronization error $S y n_{x}$ first decreases, then increases, and finally tends to infinity with the increase in noise intensity $D$. In other words, the noise with appropriate intensity enhances the consensus of FOMAS (2). That is, the effect of 'noise-induced synchronization' appears. At the same time, the black circle in Figure 11c represents the optimal noise intensity to minimize the synchronization position error under each order. It can be seen that the optimal noise intensity does not have a monotonic linear relationship with respect to the order. In addition, it can be seen from Figure $11 \mathrm{~d}$ that, with appropriate fractional-order, the average velocity synchronization error $S y n_{v}$ also has a similar 'noise-induced synchronization' effect. 

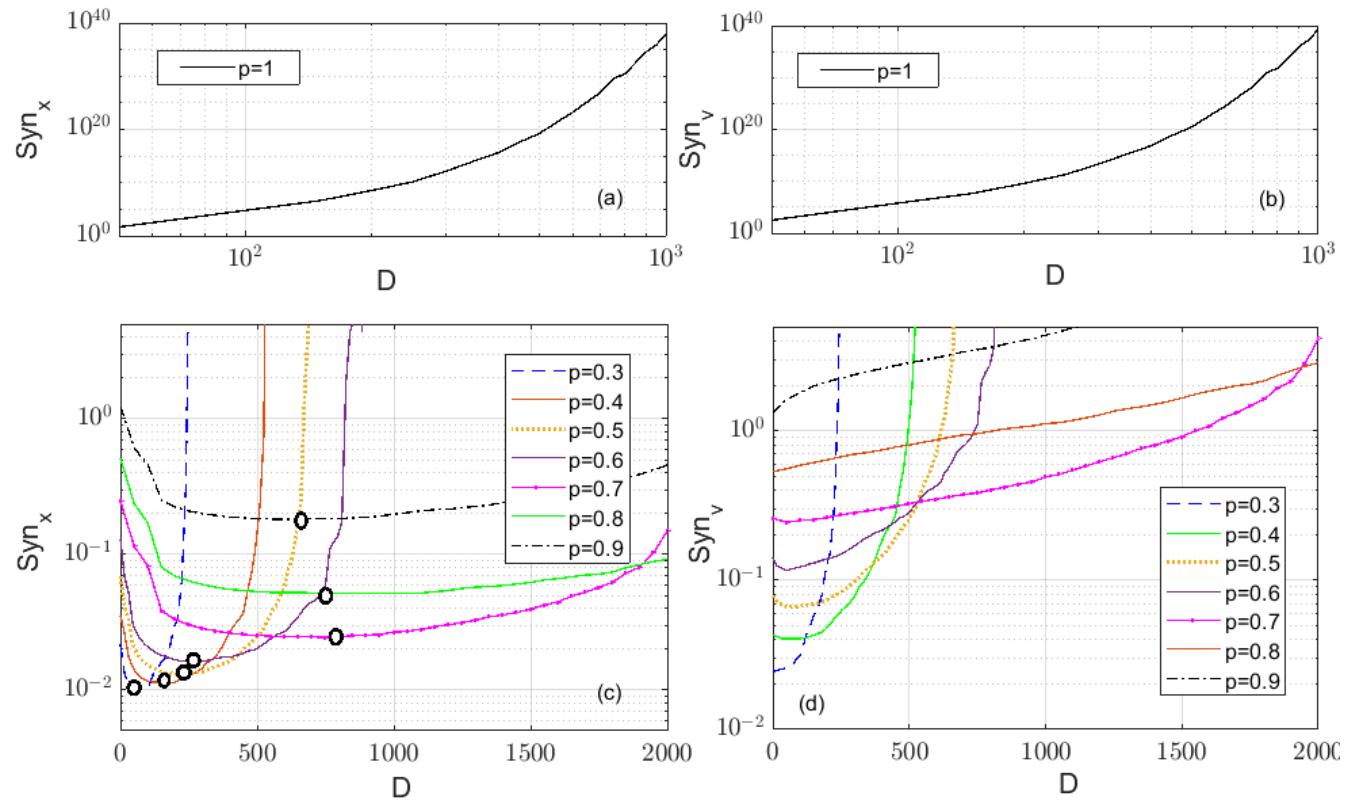

Figure 11. The average position/velocity synchronization error $\operatorname{Syn} n_{x}(\mathbf{a}, \mathbf{c})$ and $\operatorname{Syn}_{v}(\mathbf{b}, \mathbf{d})$ curves of noise intensity $D$ with different fractional-orders $p$ (bounded uniform noise).

When $\xi(t)$ is another bounded noise, a Gaussian noise controlled by a cosine function $D \cos (n(t)),(n(t)$ is a Gaussian noise with a zero mean and an intensity of 1$)$, Figure 12 shows the average position synchronization error $S y n_{x}$ and average velocity synchronization error $S y n_{v}$ curves of noise intensity D, of FOMAS (2) with different fractional orders $p$. It can be seen that the results obtained in Figure 12 are basically consistent with those in Figure 11. The two types of bounded noises, uniform noise and Gaussian noise bounded by cosine function, may both promote the consensus of the presented double-integral MAS in the fractional-order case, but does not promote the consensus of the integer-order system.
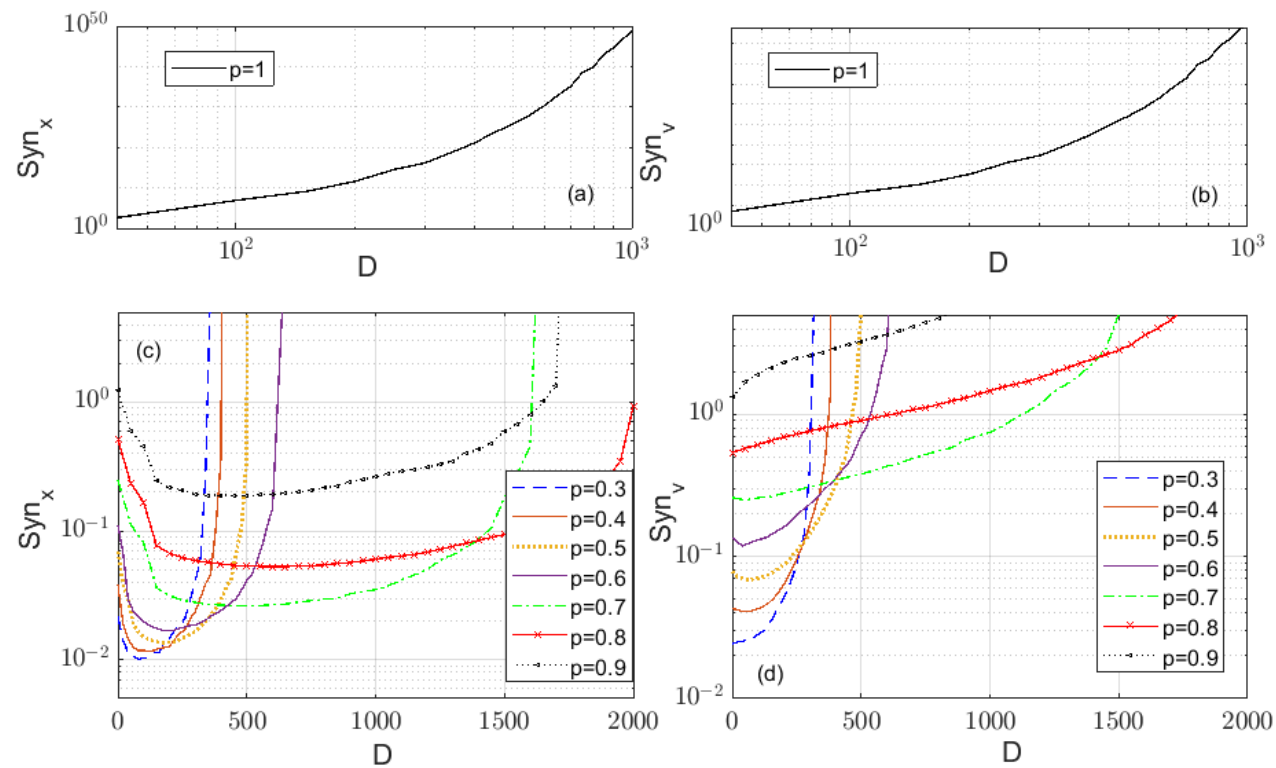

Figure 12. The average position synchronization error $\operatorname{Syn} n_{x}(\mathbf{a}, \mathbf{c})$ and average velocity synchronization error $S_{y} n_{v}(\mathbf{b}, \mathbf{d})$ curves of noise intensity $D$ with different fractional-orders $p$ (Gaussian noise controlled by cosine function).

Secondly, we analyze the influence of other parameters on the consensus of the presented FOMAS (2), in which the common noise $\xi(t)$ is still the bounded uniform noise 
in Figure 10a. Figure 13a shows the average position synchronization error $S y n_{x}$ curves of the noise intensity $D$ of the fractional-order multi-agent system (1) of $p=0.5$ with different position coupling strengths $c$, and the other parameters are $a=1, d=0.1$. It can be seen that, for small noise intensity $D$, the larger the position coupling strength $c$, the smaller the position synchronization error, which is the same as the case without noise; however, the opposite phenomenon occurs as the noise intensity increases that is, the greater the position coupling strength $c$, the greater the position synchronization error and the weaker the position consistency of the system.
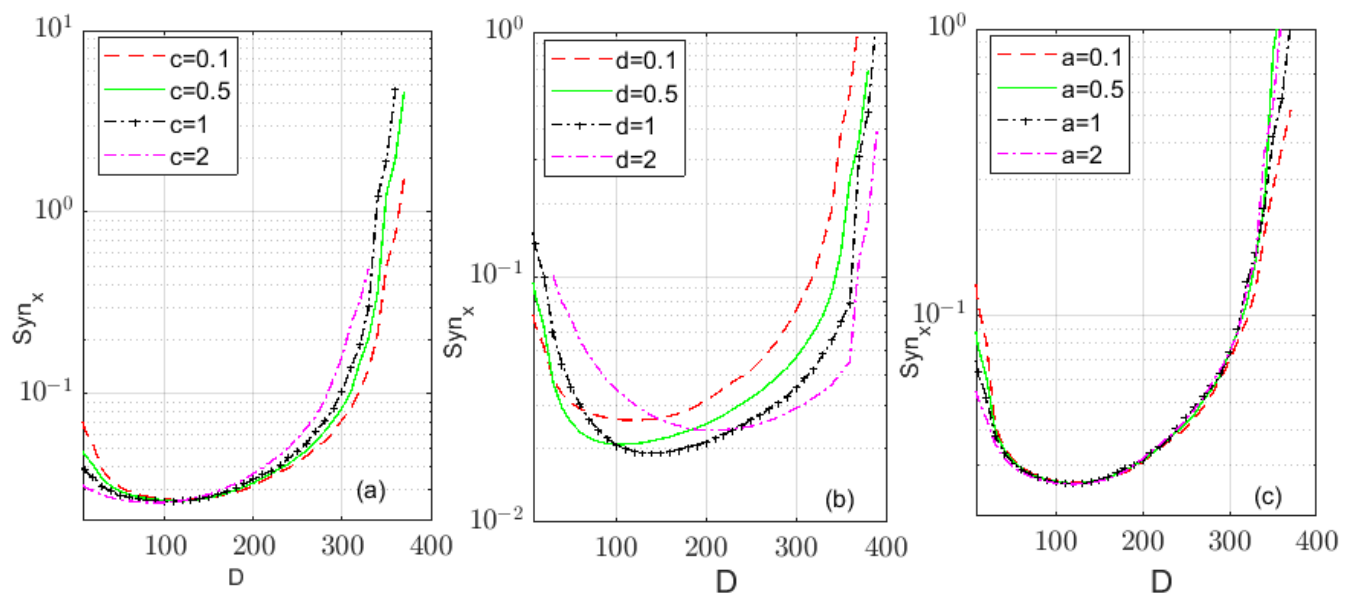

Figure 13. The average position synchronization error $S y n_{x}$ curves of noise intensity $D$ with different $a(\mathbf{a}), c(\mathbf{b})$, and $d(\mathbf{c})$.

Similarly, Figure 13b shows the average position synchronization error $S y n_{x}$ curves of noise intensity $D$ of the FOMAS (2) of $p=0.5$ with different velocity coupling strengths $d$, and the other parameters are $a=1, c=0.1$. It can be seen that the influence of velocity coupling strength $d$ on position synchronization error is just the opposite to that of position coupling strength $c$ : when the noise intensity is small, the smaller the velocity coupling strength $d$ is, the smaller the position synchronization error is, and the higher the position consistency of the system is; with the increase in noise intensity, it gradually becomes that the larger the velocity coupling strength $d$, the smaller the synchronization error and the higher the position consistency of the system.

Figure $13 c$ shows the average position synchronization error $S y n_{x}$ curves of noise intensity $D$ of the FOMAS (2) of $p=0.5$ with different potential field parameters $a$, and the other parameters are $c=0.1, d=0.1$. It can be seen that when the noise intensity is low, the larger the potential field parameter $a$ (the faster the output decays with time), the smaller the synchronization error and the higher the position consistency of the system; however, with the increase in noise intensity, the influence of the potential field parameter $a$ on position synchronization error also shows a reversal phenomenon such as the coupling strengths $c$ and $d$. That is to say, the system parameters $a, c, d$ have different effects on the system consensus under the action of noise with different intensities.

At last, we set the parameters as $a=1, c=-0.1$, and $d=-0.1$, the average position synchronization error $S y n_{x}$ curves of noise intensity $D$ of FOMAS (2) with different order $p$ are given in Figure 14, in which the common noise $\xi(t)$ is still the bounded uniform noise in Figure 10a. For this FOMAS, from Theorem 1 in Section 2.1, we know that in the absence of noise, the system with order $p<0.5$ can achieve synchronization, while the system with order $p \geq 0.5$ cannot. However, in the presence of noise, it can be seen from Figure 14 that FOMAS (2) with fractional order has the effect of 'noise-induced synchronization'. For the FOMAS (2) with $p=0.6$ and 0.7 , when the noise intensity increases to a certain value, the position synchronization error decreases significantly and is far less than 0.1 . That is to say, due to the synergistic and nonlinear effect of the bounded noise and fractional-order systems, the system achieves synchronization instead. For example, Figure 15a,b show the 
curves of the position and velocity of each agent as a function of time of a fractional-order multi-agent system $(p=0.6)$ in the absence of noise, Figure $15 c$,d show the curves of the position and velocity of each agent as a function of time of the fractional-order multi-agent system ( $p=0.6)$ in the presence of noise when the noise intensity $D=20$. It can be seen that under the action of noise, each agent realizes the synchronization of position and velocity with the increase in time $t$. This also verifies 'noise-induced synchronization' effect of the fractional-order system.

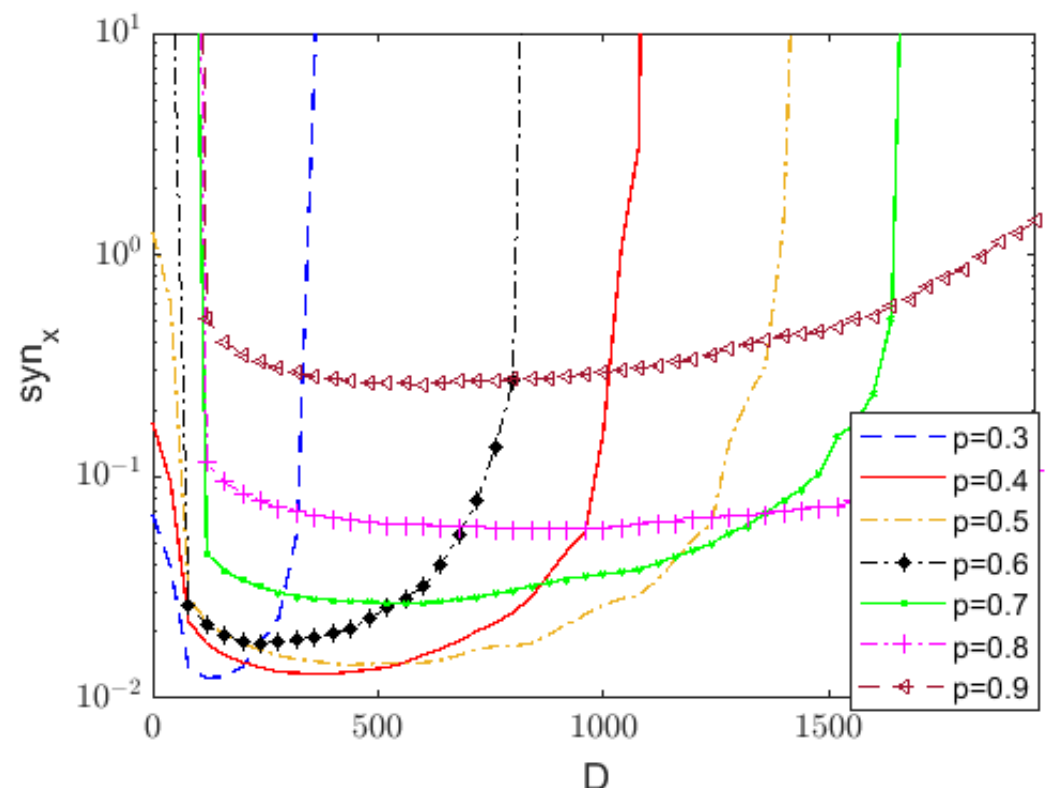

Figure 14. The average position synchronization error $S y n_{x}$ curves of noise intensity $D$ with different fractional-orders $p$ (bounded uniform noise, $a=1, c=-0.1$, and $d=-0.1$ ).
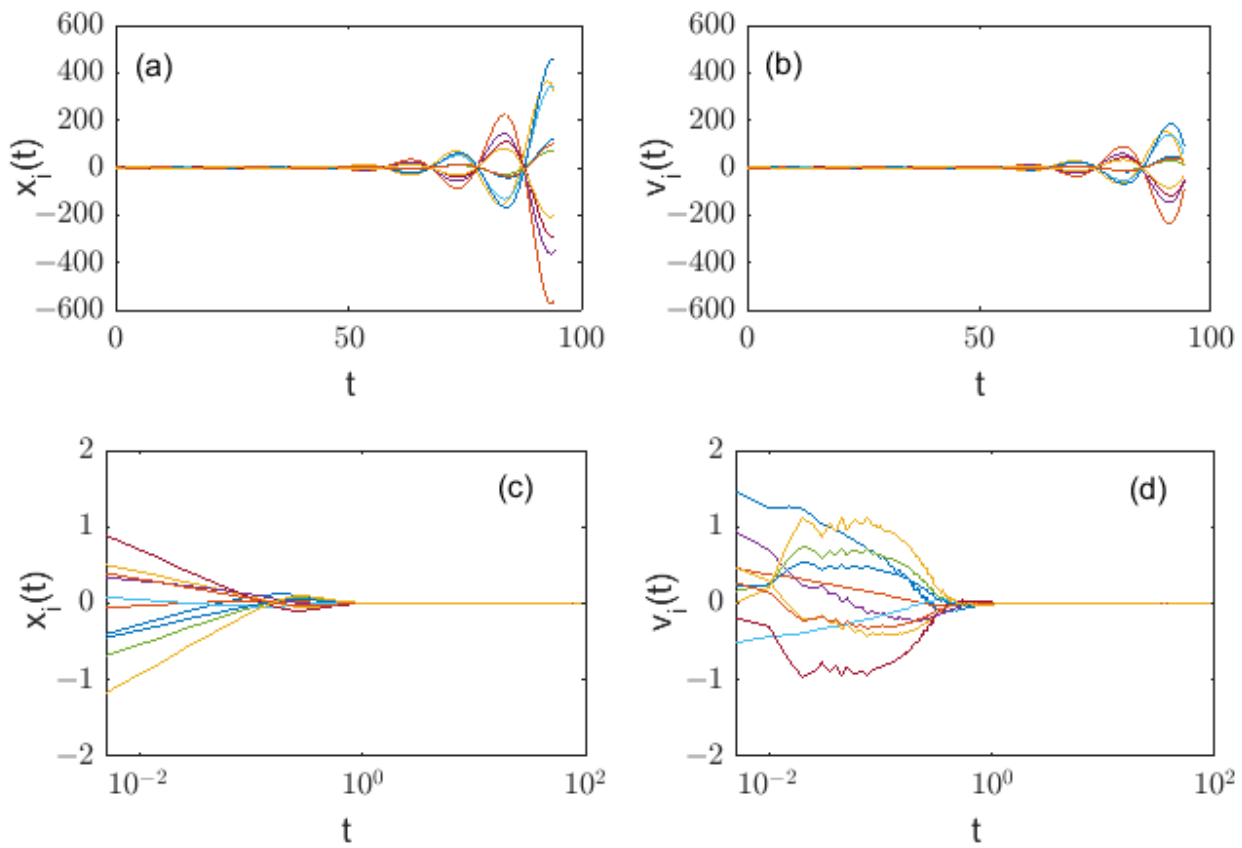

Figure 15. The curves of the position and velocity of each agent as a function of time of FOMAS (2) in the absence of noise $(\mathbf{a}, \mathbf{b})$ and in the presence of noise $(\mathbf{c}, \mathbf{d})(p=0.6)$.

\subsection{The Influence of Unbounded Noise on System Consensus}

When common noise $\xi(t)$ is an unbounded Gaussian noise (Figure 10c), Figure 16 shows the average position synchronization error $\operatorname{Syn}_{x}$ and average velocity synchroniza- 
tion error $S y n_{v}$ curves of the noise intensity D of the presented FOMAS (2) with different fractional-orders $p$. The coupling network is still the network with 10 agents given in Section 2.1, and other parameters are set as $a=1, c=0.1, d=0.1$. It can be seen that, for both the integer-order system and the fractional-order system, the average synchronization errors of position and velocity increase and tend to infinity with the increase in noise intensity D, and the 'noise-induced synchronization' effect disappears.

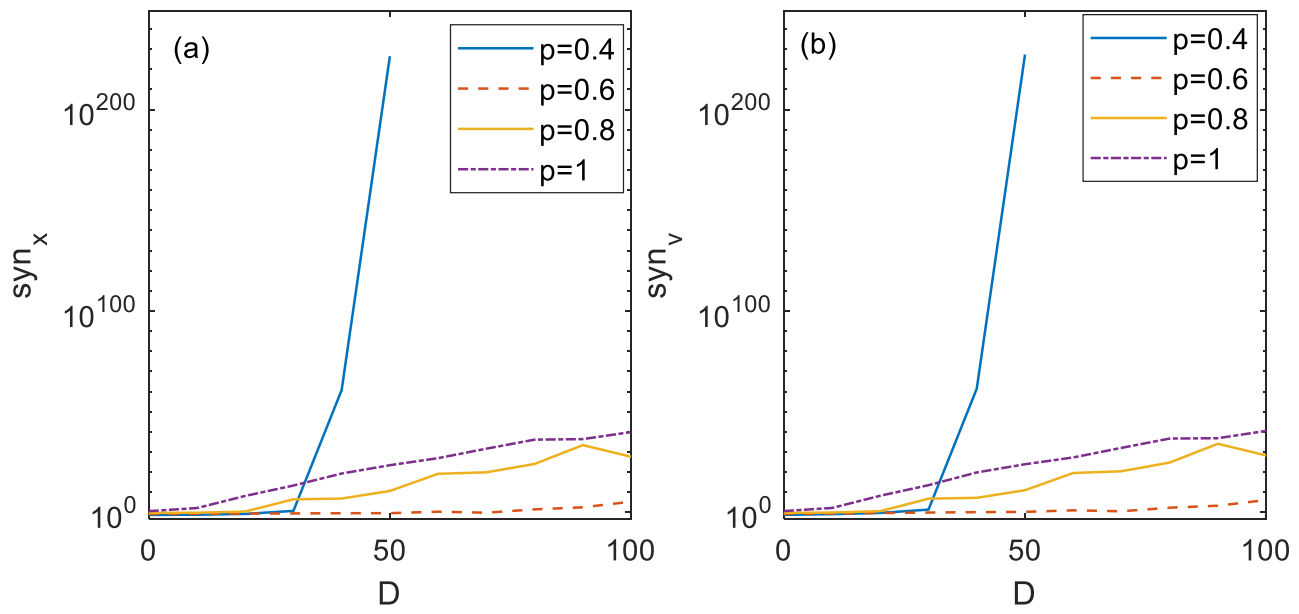

Figure 16. The average position/velocity synchronization error $S y n_{x}(\mathbf{a}) / S y n_{v}(\mathbf{b})$ curves of noise intensity $D$ of FOMAS (2) with different fractional-orders $p$ (unbounded Gaussian noise).

\section{Conclusions}

In recent years, modeling methods and theories of fractional-order dynamic systems based on the definition of fractional calculus have gradually become a new research topic in many fields such as mathematics, physics, materials, systems, biology, and economics, and have attracted the extensive attention of scholars in many fields. As a common dynamic system, the classical integer-order MASs and their consensus/synchronization behaviors have been widely studied. Very recently, scholars have gradually begun to study the FOMASs and their dynamic properties. However, there is little literature on the consensus of FOMASs under noise disturbance, which is the motivation of the study presented in this paper. In this paper, we consider the consensus problem of a double-integral FOMASs in a common bounded fluctuating potential. Our contribution comes from but goes beyond the following points:

(1) Several recent papers have discussed the consensus problem of the FOMASs in the absence of noise [23-28]. On the one hand, most protocol terms use the relative position information of neighboring agents [24,26], since the relative position state can be obtained more easily through localization methods [27]. In this paper, we consider the protocol term consisting of both the relative position and velocity information of neighboring agents, with the hope of drawing general conclusions from a more general perspective. On the other hand, most of the above literature only gives and verifies the consensus conditions [23-28]. In this paper, we not only give the analytical consensus conditions of the presented system in the absence of noise, but also analyze the influences of the system order and other system parameters on the consensus behavior in detail. It is found that the fractional order, coupling strength of the position and velocity, and potential parameters have different effects on the system's consensus behavior. Moreover, it is easier to achieve position and velocity consensus in the presented FOMAS than in the classical integer-order MAS. That is, the fractional-order system has a larger range of synchronization parameters.

(2) Most specifically, the influences of the system order and other system parameters on the consensus of the presented double-integral FOMASs in the presence of bounded noise are also analyzed in detail. It is found that, due to the synergistic and nonlinear 
effects of noise and fractional-order systems, common and bounded noise have a promoting effect on the consensus of the presented FOMAS, while it does not promote the consensus of the corresponding integer-order MAS. That is to say, the bounded noise with the appropriate intensity plays an optimization role for the presented FOMAS. It also shows that the fractional-order complex system under the action of noise has richer dynamic characteristics than the integer-order complex system. To our best knowledge, this paper is the first to report the positive effect of noise on the consensus of MASs.

(3) The fractional order and the system parameters (coupling strength and potential parameters) of the presented FOMAS have different effects on the system consensus behavior under the action of noise with different intensities. All of them can change the optimal noise intensity of the system to achieve complete consensus, which also shows that the fractional order, the system parameters, and noise play complementary roles in the complete consensus of the system. When the order and system parameters are controllable, the consensus of the system can be realized by adjusting these parameters; when the order and system parameters are uncontrollable, the doubleintegral FOMAS can also be synchronized by changing the noise intensity.

The results of this paper not only provide new theoretical results for fractional-order dynamical systems and complex systems, but also provide important theoretical guidance for the control of fractional-order stochastic dynamical systems.

Author Contributions: Conceptualization, X.C. and L.Z.; methodology, X.C. and L.Z.; software, L.Z.; validation, X.C., M.L. and L.Z.; formal analysis, L.Z.; investigation, X.C. and L.Z.; resources, M.L.; data curation, X.C. and L.Z.; writing-original draft preparation, X.C. and L.Z.; writing-review and editing, X.C. and L.Z.; visualization, X.C.; supervision, L.Z.; project administration, L.Z.; funding acquisition, L.Z. All authors have read and agreed to the published version of the manuscript.

Funding: This research received no external funding.

Institutional Review Board Statement: Not applicable.

Informed Consent Statement: Not applicable.

Data Availability Statement: Not applicable.

Conflicts of Interest: The author declares no conflict of interest.

\section{References}

1. Mainzer, K. Thinking in Complexity: The Complex Dynamics of Matter, Mind and Man-Kind; Springer-Verlag: New York, NY, USA, 1997.

2. Christen, K. Complexity and Criticality; Fudan University Press: Shanghai, China, 2006.

3. Yang, L.X.; Jiang, J. Complex dynamical behavior and modified projective synchronization in fractional-order hyper-chaotic complex Lu system. Chaos Soliton Fract. 2015, 78, 267-276. [CrossRef]

4. Wooldrideg, M. An Introduction to Multi-Agent Systems; Publishing House of Electronics Industry: Beijing, China, 2003.

5. Zhu, J. Stabilization and synchronization for a heterogeneous multi-agent system via harmonic control. Syst. Control Lett. 2014, 66, 1-7. [CrossRef]

6. Gambuzza, L.V.; Frasca, M. Distributed control of multi-consensus. IEEE Trans. Autom. Control 2020, 99, 2032-2044.

7. Mattioni, M. On multiconsensus of multi-agent systems under aperiodic and asynchronous sampling. IEEE Control Syst Lett. 2020, 4, 839-844. [CrossRef]

8. Panteley, E.; Loría, A. Synchronization and dynamic consensus of heterogeneous networked systems. IEEE Trans. Autom. Control 2017, 62, 3758-3773. [CrossRef]

9. Mattioni, M.; Monaco, S. Cluster partitioning of heterogeneous multi-agent systems. Automatica 2022, 138, 110136. [CrossRef]

10. Ren, W.; Beard, R.W. Consensus seeking in multiagent systems under dynamically changing interaction topologies. IEEE Trans. Autom. Control 2005, 50, 655-661. [CrossRef]

11. Jiang, F.; Xie, D.; Liu, B. Static consensus of second-order multi-agent systems with impulsive algorithm and time-delays. Neurocomputing 2017, 223, 18-25. [CrossRef]

12. Cacace, F.; Mattioni, M.; Monaco, S.; Ricciardi-Celsi, L. Topology-induced containment for general linear systems on weakly connected digraphs. Automatica 2021, 131, 109734. [CrossRef] 
13. Gambuzza, L.V.; Frasca, M.; Sorrentino, F.; Pecora, L.M.; Boccaletti, S. Controlling symmetries and clustered dynamics of complex networks. IEEE Trans. Netw. Sci. Eng. 2020, 8, 282-293. [CrossRef]

14. Cristofaro, A.; Mattioni, M. Hybrid consensus for multi-agent systems with time-driven jumps. Nonlinear Anal. Hybrid Syst. 2021, 43, 101113. [CrossRef]

15. Barkai, G.; Mirkin, L.; Zelazo, D. On Sampled-Data Consensus: Divide and Concur. IEEE Control Syst. Lett. 2021, 6, 343-348. [CrossRef]

16. Yu, W.; Chen, G.; Ren, W.; Kurths, J.; Zheng, W.X. Distributed higher order consensus protocols multi-agent dynamical systems. IEEE Trans. Circuits Syst. I Regul. Pap. 2011, 58, 1924-1932. [CrossRef]

17. Tang, Y.; Deng, Z.; Hong, Y. Optimal Output Consensus of High-Order Multiagent Systems With Embedded Technique. IEEE Trans. Cybern. 2018, 49, 1768-1779. [CrossRef] [PubMed]

18. Xu, Y.; Wang, J.; Zhang, Y.; Xu, Y. Event-triggered bipartite consensus for high-order multi-agent systems with input saturation Neurocomputing 2019, 379, 284-295. [CrossRef]

19. Ni, J.; Wen, C.; Zhao, Y. Fixed-time leader-follower quantized output consensus of high-order multi-agent systems over digraph. Inf. Sci. 2021, 587, 408-434. [CrossRef]

20. Podlubny, I. Fractional Differential Equations: An Introduction to Fractional Derivatives, Fractional Differential Equations, to Methods of Their Solution and Some of Their Applications; Elsevier: Amsterdam, The Netherlands, 1999.

21. Almatroud, A.O. Extreme Multi stability of a Fractional-Order Discrete-Time Neural Network. Fractal Fract. 2021, 5, 202. [CrossRef]

22. Baskonus, H.M.; Ciancio, A. New Challenges Arising in Engineering Problems with Fractional and Integer Order. Fractal. Fract. 2021, 5, 35. [CrossRef]

23. Zhu, W.; Li, W.; Zhou, P.; Yang, C. Consensus of fractional-order multi-agent systems with linear models via observer-type protocol. Neurocomputing 2016, 230, 60-65. [CrossRef]

24. Yu, W.; Li, Y.; Wen, G.; Yu, X.; Cao, J. Observer Design for Tracking Consensus in Second-Order Multi-Agent Systems: Fractional Order Less Than Two. IEEE Trans. Autom. Control 2016, 62, 894-900. [CrossRef]

25. Yu, Z.; Jiang, H.; Hu, C.; Yu, J. Necessary and Sufficient Conditions for Consensus of Fractional-Order Multiagent Systems via Sampled-Data Control. IEEE Trans. Cybern. 2017, 47, 1892-1901. [CrossRef] [PubMed]

26. Liu, H.; Xie, G.; Gao, Y. Consensus of fractional-order double-integrator multi-agent systems. Neurocomputing 2019, 340, 110-124. [CrossRef]

27. Wang, A.; Liao, X.; Dong, T. Fractional-order follower observer design for tracking consensus in second-order leader multi-agent systems: Periodic sampled-based event-triggered control. J. Frankl. Inst. 2018, 355, 4618-4628. [CrossRef]

28. Hu, T.; Park, J.H.; He, Z.; Zhang, X.; Zhong, S. State-based event-triggered consensus strategy for Takagi-Sugeno fuzzy fractionalorder multiagent systems with switching topologize. ISA Trans. 2021. [CrossRef] [PubMed]

29. Cheng, L.; Wang, Y.; Hou, Z.-G.; Tan, M.; Cao, Z. Sampled-data based average consensus of second-order integral multi-agent systems: Switching topologies and communication noises. Automatica 2013, 49, 1458-1464. [CrossRef]

30. Sun, W.; Lü, J.J.; Yu, X.; Chen, S. Second-order consensus of multi-agent systems with noise. IET Control Theory Appl. 2014, 8, 2026-2032. [CrossRef]

31. Ming, P.; Liu, J.; Tan, S.; Wang, G.; Shang, L.; Jia, C. Consensus stabilization of stochastic multi-agent system with Markovian switching topologies and stochastic communication noise. J. Frankl. Inst. 2015, 352, 3684-3700. [CrossRef]

32. Chen, J.; Ling, Q. A robust quantized consensus protocol for discrete-time multi-agent systems with additive noise. ISA Trans. 2018, 86, 29-38. [CrossRef] [PubMed]

33. Wu, Y.; Gong, Y.; Wang, Q. Noise-induced synchronization transitions in neuronal network with delayed electrical or chemical coupling. Eur. Phys. J. B 2014, 87, 198-1-198-6. [CrossRef]

34. Zhou, C.; Kurths, J.; Hu, B. Array-Enhanced Coherence Resonance: Nontrivial Effects of Heterogeneity and Spatial Independence of Noise. Phys. Rev. Lett. 2001, 87, 98101. [CrossRef]

35. Wang, J.; Zhang, J.; Yuan, Z.; Zhou, T. Noise-induced switches in network systems of the genetic toggle switch. BMC Syst. Biol. 2007, 1, 50. [CrossRef] [PubMed]

36. Sun, X.; Lu, Q.; Kurths, J. Correlated noise induced spatiotemporal coherence resonance in a square lattice network. Phys. A Stat. Mech. Its Appl. 2008, 387, 6679-6685. [CrossRef]

37. Maritan, A.; Banavar, J.R. Chaos, noise, and synchronization. Phys. Rev. Lett. 1994, 72, 1451. [CrossRef]

38. Kiss, I.Z.; Zhai, Y.; Hudson, J.L.; Zhou, C.; Kurths, J. Noise enhanced phase synchronization and coherence resonance in sets of chaotic oscillators with weak global coupling. Chaos Interdiscip. J. Nonlinear Sci. 2003, 13, 267-278. [CrossRef] [PubMed]

39. Lai, L.; Zhang, L.; Yu, T. Collective behaviors in globally coupled harmonic oscillators with fluctuating damping coefficient. Nonlinear Dyn. 2019, 97, 2231-2248. [CrossRef]

40. Zhong, S.; Zhang, L. Noise effect on the signal transmission in an underdamped fractional coupled system. Nonlinear Dyn. 2020, 102, 2077-2102. [CrossRef]

41. Pecora, L.M.; Carroll, T.L. Master stability functions for synchronized chaos in arrays of oscillators. In Proceedings of the IEEE International Symposium on Circuits \& Systems, Monterey, CA, USA, 31 May-3 June 1998. 